\title{
Role of 18F-FDG PET Scan in Parapharyngeal Space Tumors: Report of a Rare Case and Review of the Literature
}

\author{
Sahel OA ${ }^{1^{*}}$, Biyi $\mathrm{A}^{1}$, Ennouali $\mathrm{H}^{2}$, Benameur $\mathbf{Y}^{1}$, Bouyallaoui $\mathbf{H}^{1}$, and Doudouh $\mathrm{A}^{1}$ \\ ${ }^{1}$ Department of Nuclear Medicine, Military Hospital of Instruction Mohamed V, University Med V-Rabat, Morocco \\ ${ }^{2}$ Department of Radiology, Military Hospital of Instruction Mohamed V, University Med V-Rabat, Morocco
}

\begin{abstract}
Background: Tumors of the parapharyngeal space (PPS) are rare and account for only $0.5 \%$ of head and neck neoplasms. Approximately $80 \%$ of these tumors are benign lesions, yet they represent a formidable diagnostic and treatment challenge. They are difficult to diagnose because they have few symptoms, therefore, CT and MRI are essential to delineate the tumor extent, intracranial involvement and relationship to the adjacent vital structures.

Case presentation: We report the case of a 36-year-old male who presented with the sudden onset of a pharyngeal cavity mass. CT head and neck scan and MRI showed a left paraphayngeal mass occupying the prestyloid parapharyngeal space extending to poststyloid. Transorally biopsy revealed a well differenced squamous cell carcinoma. A FDG PET/CT was performed and revealed outside the known tumor, vertebral metastasis of the cervical and thoracic spine, a right collarbone metastasis and pulmonary nodules. It was decided to manage this metastatic squamous cell carcinoma with chemotherapy.

Conclusion: Parapharyngeal squamous cell carcinomas are extremely rare. Imaging helps in planning the surgical procedure. Radionuclide imaging is complimentary to radiological imaging and provides specific information about the tumor's functional and molecular characteristics. This case of squamous cell carcinoma presents several unusual features: the scarcity of squamous cell carcinoma in the paraphayngeal space and faraway metastasis in this type of tumor. Moreover the role of FDG PET/CT have been exceptionally described in the literature.
\end{abstract}

Keywords: Parapharyngeal space tumors; Squamous cell carcinoma; Diagnostic imaging; Bone metastasis; FDG-PET

\section{Background}

Parapharyngeal space (PPS) tumors are rare, accounting for $0.5 \%$ of all neoplasms of head and neck. PPS tumors may be primary, metastatic or a direct extension of tumors from the adjacent spaces. The frequency of benign and malignant tumors is about $80 \%$ and $20 \%$, respectively. Most of them originate from the salivary gland (40-50\%), followed by a neurogenic etiology and the remainder are a broad spectrum of miscellaneous tumors [1-7].

The PPS is a lateral pharyngeal region. It is divided by muscular aponeurotic partition into an anterior (prestyloid) muscular compartment and a posterior (poststyloid) neurovascular compartment [1,3-7]. Imaging is crucial for the assessment of PPS tumors. Surgery is performed based on information provided by these diagnostic modalities.

This article will present a case of left parapharyngeal squamos cell carcinoma with unusual spinal metastases, and the potential role of FDG PET/CT will be illustrated.

\section{Case Presentation}

A 36-year-old male patient who showed up in April 2016 with 3 months' history of left side facial paralysis, left trismus, difficulty in swallowing and otological symptoms: otorrhea and otorrhagia. For the last month, he had developed a pharyngeal cavity mass. CT scan head and neck showed well defined heterogeneously enhancing left parapharyngeal solid mass $(32 \times 50 \times 62 \mathrm{~mm})$ with multiple area of necrosis, bulging into nasopharynx with extension to pharyngeal mucosal surface.

The mass overwhelms the tympanic cavity and spreads to the ipsilateral external auditory meatus and pterygoid muscle. It causes the lysis of base of skull and the posterior walls of the left sphenoidal and cavernous sinuses, with endocranial extension. This aspect suggested either the diagnosis of schwannoma or glomus vegale, so we decided on MRI. Head and neck MRI confirmed the left parapharyngeal pear shaped mass occupying the prestyloid parapharyngeal space extending to poststyloid space bulging into the nasopharynx, likely representing schwannoma (Figure 1).

Transorally biopsy of the roof of mouth revealed a well differenced squamous cell carcinoma: round and polygonal cells with large pleomorphic nuclei, aggregates of spindle shaped cells have been seen entrapped within a cellular, hyaline stroma, areas of necrosis and desmoplastic reaction present. Immunostaining was positive for cytokeratin.

Bone scintigraphy with radio bi-phosphonates was immediately requested to look for bone locations. Our patient received an intravenous injection of $680 \mathrm{MBq}$ of ${ }^{99 \mathrm{~m}} \mathrm{Tc}-\mathrm{MDP}$ and whole body scanning was done after 2 h 30 min delay. It showed normal and symmetrical uptake of ${ }^{99 m} \mathrm{mc}-\mathrm{MDP}$ on the whole skeleton without suspected secondary bone localization (Figure 2). Chest and abdominal computed tomography

*Corresponding author: Sahel OA, Department of Nuclear Medicine, Military Hospital of Instruction Mohamed V, University Med V-Rabat, Morocco, Tel: +0021262841784; Fax: +0021237563436; E-mail: omaraitsahel85@gmail.com

Received October 27, 2016; Accepted January 25, 2017; Published January 27 2017

Citation: Sahel OA, Biyi A, Ennouali $\mathrm{H}$, Benameur $\mathrm{Y}$, Bouyallaoui $\mathrm{H}$, et al (2017) Role of 18F-FDG PET Scan in Parapharyngeal Space Tumors: Report of a Rare Case and Review of the Literature. J Cancer Sci Ther 9: 321-324 doi: 10.4172/1948-5956.1000436

Copyright: $\odot 2017$ Sahel OA, et al. This is an open-access article distributed under the terms of the Creative Commons Attribution License, which permits unrestricted use, distribution, and reproduction in any medium, provided the original author and source are credited. 
Citation: Sahel OA, Biyi A, Ennouali H, Benameur Y, Bouyallaoui H, et al. (2017) Role of 18F-FDG PET Scan in Parapharyngeal Space Tumors: Report of a Rare Case and Review of the Literature. J Cancer Sci Ther 9: 321-324. doi: 10.4172/1948-5956.1000436

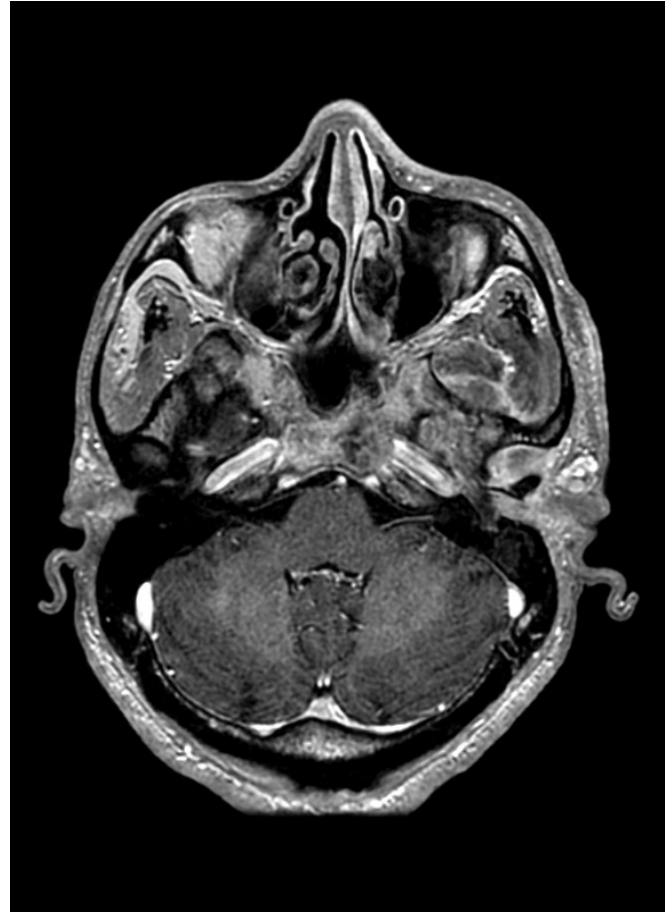

Figure 1: Head and neck MRI in axial slices showing a left parapharyngeal infiltrating process extended to the ipsilateral infra temporal fossa; isointense T1 and T2 enhanced heterogeneously after gadolinium injection.

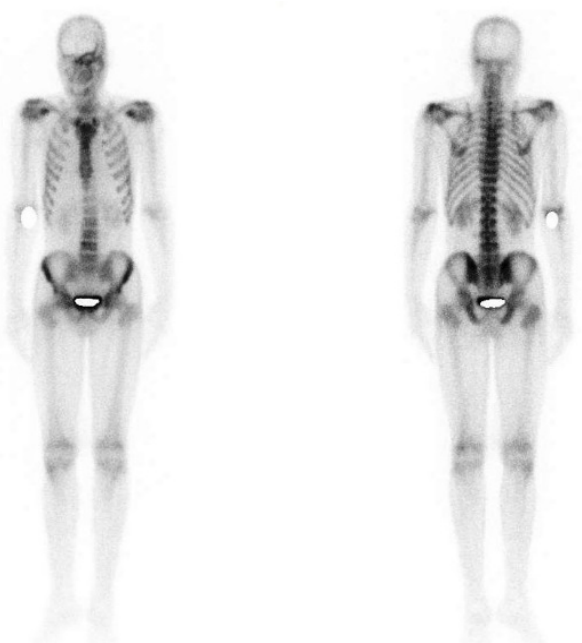

Figure 2: Whole body scintigraphy showing normal and symmetrical uptake of $99 \mathrm{mTC}-\mathrm{MDP}$ on the whole skeleton without suspected secondary bone localization.

(CT) were carried out for the work up of the spread of the squamous cell carcinoma. It showed two suspected left pulmonary nodules: the first, measuring $11 \mathrm{~mm}$, was localized in the upper lobe; the second, measuring $12 \mathrm{~mm}$, in the lingula. A FDG PET/CT was performed to characterize the pulmonary nodules. It revealed outside the known tumor, an increased uptake of ${ }^{18} \mathrm{~F}-\mathrm{FDG}$ at the vertebral column from $\mathrm{C} 1$ to $\mathrm{T} 10$ and the right collarbone. It also indicated an intense ${ }^{18} \mathrm{~F}-\mathrm{FDG}$ activity in the left higher lobe (standard uptake value=10.0), corresponding to pulmonary nodules (Figure 3). The degree of activity was highly suspicious of malignancy; suggesting pulmonary metastasis nodules from the primary squamous cell carcinoma of the parapharyngeal space. Following multidisciplinary consultation, it was decided to manage this squamous cell carcinoma with intracerebral extension, pulmonary and bone metastasis by chemotherapy with docetaxyl and cisplatin [8].

\section{Discussion}

The parapharyngeal space is in the shape of an inverted pyramid on pedestal [9]. The base is formed by the greater wing of the sphenoid at the skull base. The apex is at the level of the greater cornu of the hyoid bone. The medial wall is composed of the superior constrictor muscle. The prevertebral fascia forms the posterior wall. The lateral wall from an anteroposterior direction is formed by the medial pterygoid muscle, vertical ramus of the mandible, the deep lobe of the parotid, and the posterior belly of the digastrics muscle, respectively. The parapharyngeal space is divided into prestyloid and psotstyloid spaces. The prestyloid space contains the medial and the lateral pterygoid muscles, the ramus and condyle of the mandible, the deep lobe of the parotid gland, the maxillary artery, and branches of V3. The retrostyloid space contains the carotid sheath, the cervical sympathetic trunk, cranial nerves IX, $\mathrm{X}, \mathrm{XI}$, and XII, and multiple lymph nodes. Because the base, lateral and posterior walls of the parapharyngeal space are bony, tumors tend to grow medially. Hence, the most common physical sign is a medial and inferior displacement of the soft palate and tonsillar fossa.

Tumours of the parapharyngeal space PPS remain clinically silent for a long time till they attain a sufficiently large size to produce a palpable or visible lump in the oral cavity and neck. The symptoms of PPS tumors are multiple and relate to the prestyloid-poststyloid localization, they include frequent foreign body sensation, difficult deglutition, ontological symptoms, and hoarseness. Clinical manifestations of PPS tumors are a mass in the oropharynx, a neck mass, cranial nerve deficit and Eustachian tube obstruction [2,4,6-8].

In a multicentric meta-analysis of 1143 parapharyngeal space tumors presented in 17 studies, Riffat et al. showed that the most prestyloid tumors arise from the salivary glands, with a total of 15 different histological types reported in the cumulative series [10].

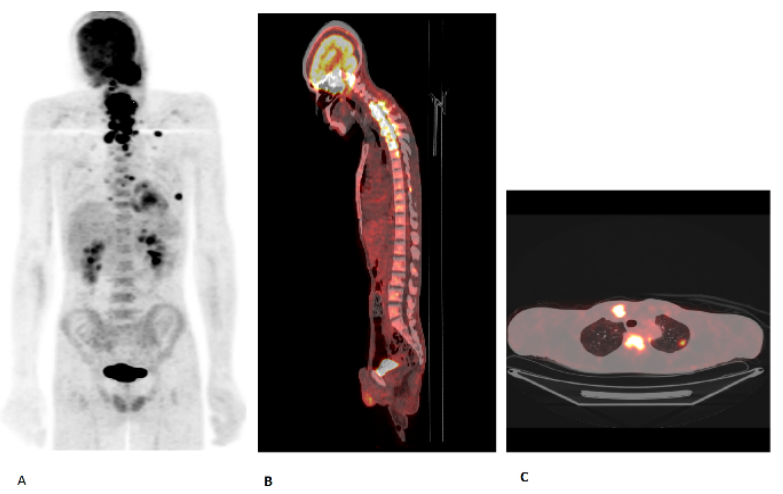

Figure 3: 18F-FDG PET/CT. A: PET/CT (MIP slice) performed at staging, showed pathological foci on the left parapharyngeal space, associated to pathological foci of vertebral column extended from C1 to T10 and a right collarbone increased uptake of FDG. It also showed increased pathological uptake of $18 \mathrm{~F}-\mathrm{FDG}$ in the pulmonary nodules. B: sagittal slice showing increased uptake of 18F-FDG on the vertebral column. C: PET/CT in axial slice at pulmonary parenchymal window showing an increased uptake in the pulmonary nodule. 
Citation: Sahel OA, Biyi A, Ennouali H, Benameur Y, Bouyallaoui H, et al. (2017) Role of 18F-FDG PET Scan in Parapharyngeal Space Tumors: Report of a Rare Case and Review of the Literature. J Cancer Sci Ther 9: 321-324. doi: 10.4172/1948-5956.1000436

Parapharyngeal squamous cell carcinomas are extremely rare, and only a few cases have been described in the literature. It is usually seen as a metastatic nodal disease or less commonly as an extension of deep lobe parotid tumor into the parapharyngeal space. Som et al. [11] and Maran et al. [12] have reported an incidence of $14 \%$ and $17 \%$ respectively. Rarely, primary PPS tumours can arise from lateral pharyngeal wall or minor salivary gland tissue of PPS (SCC comprises $1.2 \%$ of minor salivary gland epithelial neoplasms) $[13,14]$. The tumor in our case probably originated anew from minor salivary glands of the parapharyngeal space or from lateral pharyngeal wall.

CT scan and MRI are important diagnostic tools in tumors of parapharyngeal space. These helps in determining the extent of disease, local spread and help to some extent in determining the type of tumor. Contrast enhancement is seen in vascular and neurogenic tumors. Presence of intact fat plane helps in distinguishing benign tumors from malignant ones. Extension of tumors from the deep lobe of a parotid gland is distinguishable from tumor arising anew in parapharyngeal space by a fine translucent line representing the compressed layer of fibroadipose tissue between the tumor and deep lobe of parotid [15]. MRI has been shown to be superior to computed tomography in the investigation of parapharyngeal space tumors [16,17]. Computed Tomography (CT) and Magnetic Resonance Imaging (MRI) are the cornerstone in the initial evaluation. Radionuclide imaging is complimentary to radiological imaging and provides specific information about the tumor's functional and molecular characteristics. Positron emission tomography (PET) has a higher sensitivity than single-photon emission computed tomography (SPECT) and provides images of better resolution [18].

In addition, FDG-PET has been known as a useful method in detecting bone metastasis in pre-operative patients or in post-operative follow-up patients [19-21]. On the other hand, conventional bone scintigraphy (BS) is an established imaging modality as a first choice for detecting bone metastasis, but also known for its drawback in detecting pure osteolytic bone metastases [19,20,22].

MRI and ${ }^{18}$ F-FDG PET can detect malignant marrow involvement early during the disease before identifiable bone destruction or reactive osteoblastic changes occur and, thus, may precede CT and BS in identifying the presence of malignant bone involvement. Although 18F-fluoride uptake depends on regional blood flow and osteoblastic activity, like ${ }^{99 \mathrm{~m}} \mathrm{Tc}-\mathrm{MDP}$, the better spatial resolution of PET and the favorable pharmacokinetic characteristics of 18F-fluoride make 18F-fluoride PET a more sensitive modality for detecting both lytic and blastic lesions [23]. In our case BS and CT misdiagnosed the column metastases; only FDG-PET was positive.

Fine needle aspiration cytology is the modality of choice for obtaining biopsy sample for diagnosis [24].

Surgery is the mainstay of treatment in parapharyngeal tumors. The surgical approach chosen should facilitate complete tumor extirpation with minimal morbidity. The conventional surgical approaches described for the parapharyngeal tumors are transoral, transparotid, trans-cervical with or without mandibulectomy and midline transmandibular-oropharyngeal approaches. The correct choice between them depends upon the accurate information on mass size and location, its relationship with the surrounding vessels and nerves and its nature [25].

\section{Conclusion}

Parapharyngeal squamous cell carcinomas are extremely rare.
Computed Tomography (CT) and Magnetic Resonance Imaging (MRI) are the cornerstone in the initial evaluation. Radionuclide imaging is complimentary to radiological imaging and provides specific information and higher sensitivity. Surgery is the mainstay of treatment in parapharyngeal tumors.

\section{References}

1. Batsakis JG, Sneige N (1989) Parapharyngeal and retropharyngeal space diseases. Ann Otol Rhinol Laryngol 98: 320-321.

2. Carrau R, Myers E, Johnson J (1990) Management of tumors arising in the parapharyngeal space. Laryngoscope 100: 583-589.

3. Ducic Y, Oxford L, Pontius AT (2006) Transoral approach to the superomedial parapharyngeal space. Otolaryngol Head Neck Surg 134: 466-470.

4. Khafif A, Segev Y, Kaplan DM, Gil Z, Fliss DM (2005) Surgical management of parapharyngeal space tumors: a 10-year review. Otolaryngol Head Neck Surg 132: $401-406$

5. Malone JP, Agrawal A, Schuller DE (2001) Safety and efficacy of transcervical resection of parapharyngeal space neoplasms. Ann Otol Rhinol Laryngol 110 1093-1098.

6. Myers EN, Johnson JT, Curtin HG (2003) Tumors of parapharyngeal space Cancer Head Neck 22: 511-513.

7. Work WP, Hybels RL (1974) A study of tumors of the parapharyngeal space. Laryngoscope 84: 1748-1755.

8. Allison RS, Van der Waal I, Snow GB (1989) Parapharyngeal tumours: a review of 23 cases. Clin Otolaryngol Allied Sci 14: 199-203.

9. Biller H, Som P, Lawson W (1981) Tumors of the parapharyngeal space, preoperative evaluation, diagnosis and surgical approaches. Ann Otol Rhino Laryngol 90: 3-1.

10. Riffat F, Dwivedi RC, Palme C, Fish B, Jani P5 (2014) A systematic review of 1143 parapharyngeal space tumors reported over 20 years. Oral Oncol 50: 421-430.

11. Som PM, Biller HF, Lawson W, Sacher M, Lanzieri CF (1984) Parapharyngeal space masses: an updated protocol based upon 104 cases. Radiology 153: 149-156.

12. Maran AG, Mackenzie IJ, Murray JA (1984) The parapharyngeal space. J Laryngol Otol 98: 371-380.

13. Chabot M, Fradet G, Thériault R, Morrissette YP (1996) The excision of branchial parapharyngeal cysts by transbuccal or I cervical approach. J Otolaryngol 25: 108-112.

14. Evenson JW, Cawson RA (1985) Salivary gland tumours. A Review of 2410 cases with particular reference to histological types, sites, age and sex distribution. J Path 146: 51-8

15. Work PW, Gates GA (1969) Tumours of parapharyngeal space. Otolaryngo Clin N Am 479-514.

16. Lloyd GAS, Phelps PD (1986) The demonstration of tumours of the parapharyngeal space by magnetic resonance imaging. $\mathrm{Br} \mathrm{J}$ Radiol 59: 675-683.

17. Tsushima Y, Matsumoto M, Endo K (1994) Parotid and parapharyngeal tumours; tissue characterization with dynamic magnetic resonance imaging. Br J Radiol 67: 342-345.

18. Varoquaux A, Fakhry N, Gabriel S, Garcia S, Ferretti A, et al. (2013) Retrostyloid parapharyngeal space tumors: A clinician and imaging perspective. Eur $\mathrm{J}$ of Radiol 82: 773- 782

19. Eubank WB, Mankoff DA (2005) Evolving role of positron emission tomography in breast cancer imaging. Semin Nucl Med 35: 84-99.

20. Maffioli L, Florimonte L, Pagani L, Butti I, Roca I (2004) Current role of bone scan with phosphonates in the follow-up of breast cancer. Eur J Nucl Med Mol Imaging 31: 143-148.

21. Fogelman I, Cook G, Israel O, Van der Wall H (2005) Positron emission tomography and bone metastases. Semin Nucl Med 35: 135-142. 
Citation: Sahel OA, Biyi A, Ennouali H, Benameur Y, Bouyallaoui H, et al. (2017) Role of 18F-FDG PET Scan in Parapharyngeal Space Tumors: Report of a Rare Case and Review of the Literature. J Cancer Sci Ther 9: 321-324. doi: 10.4172/1948-5956.1000436

22. Abe K, Sasaki M, Kuwabara Y, Koga H, Baba S, et al. (2005) Comparison of 18FDG-PET with $99 \mathrm{mTc}-\mathrm{HMDP}$ scintigraphy for the detection of bone metastases in patients with breast cancer. Ann Nucl Med 19: 573-579.

23. Even-Sapir E1 (2005) Imaging of malignant bone involvement by morphologic, scintigraphic, and hybrid modalities. J Nucl Med 46: 1356-1367.
24. Rodriguez-Giurana J, Rodado C, Saez M, Bassas C (2000) Giant parotid pleomorphic adenoma involving the parapharyngeal Space: report of a case. $J$ Oral Maxillofac Surg 58: 1184-1187.

25. Ahmad F, Waqar-uddin, Khan MY, Khawar A, Bangush W, et al. (2006) Management of parapharyngeal space tumours. J Coll Physicians Surg Pak 16: $7-10$. 